\title{
Research of the Membrane Separation Technology on Traditional Chinese Medicine Extraction
}

\author{
Jingya Zhang ${ }^{1}$ \\ ${ }^{1}$ Henan University of Traditional Chinese Medicine, Zhengzhou, 450008 \\ hunter2011@foxmail.com
}

Keywords: Chinese Medicine; Preparation; Membrane Separation Technology

\begin{abstract}
The membrane separation technology used in Chinese medicine extracts, namely extracts microfiltration to remove suspended solids, and then to ultra-filtrate to remove impurities macromolecules. By compared microfiltration, ultra-filtration process of investigation with traditional alcohol precipitation process, this paper holds that the adoption of a membrane separation process has significant advantages.
\end{abstract}

\section{Introduction}

After the founding of our country has been the cause of the rapid development of traditional Chinese medicine, the pharmaceutical companies from the original hand-workshop to develop into a certain scale enterprises, in 1996 the national pharmaceutical companies existing in 1059, total value of 23.54 billion RMB of Chinese medicine industry. Chinese medicine production is extracted from finishing, processing, sterilization. Preparation, packaging and production of some traditional formulations are basically using the machine, and some initial realization of mechanization and semi-mechanization of production, some of the equipment also achieved process control. However, due to institutional constraints, in 1979 before the Chinese medicine production plant owned commercial port management, technological innovation without investment, lack of research professionals in traditional Chinese medicine production equipment, so that Chinese medicine production compared with the rapid development of modern science and technology, is clearly lagging behind, more talk not on traditional Chinese medicine production process engineering.

80 years since the reform and opening up a number of medium-sized enterprises in accordance with domestic and international market requirements for products, the production environment, process equipment, made some technological innovation, developed a number of traditional Chinese medicine production process to meet the requirements of the equipment. Currently there are stand-alone devices for pharmaceutical pill equipment, granules equipment, including spray granulation, spray drying equipment; risers equipment, including continuous dynamic extraction, thin film evaporation, three-way enrichment equipment; medicine pre-treatment equipment, including turn Guochao medicine machine, drum machine, and so fried drugs; multi-process continuous production equipment, including a dynamic filter to extract a concentrate a drying equipment, penetration film evaporator continuous extraction, continuous extraction equipment, oral liquid filling line, wine filling production lines.

Membrane separation technology is developed in recent decades of separation technology, with its normal operations, the majority of non-phase change process, low energy consumption and high separation efficiency, find application in many areas, has also been used in Chinese medicine industry. There are more reports by ultra-filtration and purified extract of Chinese medicine, in order to achieve clarification, cleaning purposes.

This test intends to have a better clinical effect of compound Chinese medicine decoction, developed into granule products. With the development of Chinese medicine theory and formulation technology, the traditional water extraction and alcohol precipitation impurity has exposed some shortcomings; and the present compound in gypsum and other inorganic ingredients, but also containing active ingredients such as alkaloids, glycosides, flavonoids, phenols other 
categories, the molecular weight of less than 1000, using alcohol precipitation may make large losses. Therefore proposes the following new technology.

New processes to microfiltration are ultra-filtration and two refined alternative alcohol precipitation. Microfiltration process to deal directly with Chinese herb extractions, removal of a large number of sub-particles, particles and flocculent precipitation, ultra-filtration provides reliable pretreatment. Ultra-filtration removes liquid starch, gum, pectin, phlegmatic, proteins and other macromolecules soluble impurities.

Ultra-filtration pretreatment is the key to ensure effective operation. The most commonly used polymeric microspheres to use more dead-end filtration membrane, with the filter, the accumulation of trapped particles form a filter cake, filter resistance increases, the flux is getting smaller and smaller, it can not be long-term continuous operation. In addition, there is a narrow polymer material p $\mathrm{H}$ range, high pressure recoil regeneration technology is difficult to use other shortcomings. In this study, tubular ceramic microfiltration membrane, characterized in that the chemical stability, thermal stability and high mechanical strength; ultra-filtration process is using high unit loading area of the hollow fiber membrane.

\section{Experimental part}

Membrane and membrane module. Microfiltration membrane using the Nanjing University of Chemical Institute of Science and Technology to develop the internal pressure of the tubular ceramic membrane, an outer diameter of $12 \mathrm{~mm}$, an inner diameter of $8 \mathrm{~mm}$. Composition of $\alpha-\mathrm{Al} 2$ O3, by the support body, transition and top filter film, see the literature concerning the preparation of the film and the like. Study, when a single film tube length $500 \mathrm{~mm}$, an average pore size of the membrane $0.2 \mu \mathrm{m}$. Membrane of stainless steel, non-toxic and tasteless silicone rubber gasket is significant. UF hollow fiber ultra-filtration membrane, MWCO 50,000 membrane size is $50 \times 265$ mm.

Experimental content. Obtained by boiling the extract side, crude drug concentration of about $0.8 \mathrm{~g} / \mathrm{m} . \mathrm{l}$ take a certain amount directly to clarify the effect of the extract and process characteristics cycle microfiltration, microfiltration study; microfiltration permeate subjected to ultra-filtration, ultra-filtration impurity effect inspect properties and processes; permeate after ultra-filtration add vacuo adjuvant, etc. made of solid preparation.

Water extraction and alcohol precipitation technology and new technology comparison test of the two formulations calcium, sulfate, alkaloids, phenols and other ingredients.

Analytical method. The using of gravimetric method and qualitative testing active ingredient is good. Calcium: under acidic conditions ammonium oxalate test whether a white precipitate. Sulfate: with barium chloride test whether there is a white precipitate insoluble in hydrochloric acid. Alkaloids: bismuth potassium iodide test whether there is a reddish-brown precipitate or silicon acid test whether there is an off-white precipitate. Phenols: ferric chloride test whether there is a blue-green precipitate. Terpenes, steroids, saponins: concentrated sulfuric acid reaction with acetic anhydride (Lieberma Burchard reaction) test.

\section{Results and discussion}

Microfiltration process. Microfiltration permeate is clear and transparent, orange-red, decentralization at $15^{\circ} \mathrm{C}$. Set the number of days does not appear turbid, relatively non-perishable raw material liquid. The total solids content of the feed solution and the permeate was $3.4 \%$ and 2.6\%. By microfiltration, the liquid in the suspended impurities completely removed.

At $25^{\circ} \mathrm{C}$, filter pressure $0.12 \mathrm{MPa}$, the flow rate of flux change with time under $4 \mathrm{~m} / \mathrm{s}$ condition. Seen in 10 min later dropped from Figure 2, the flux is very slow, 150 min after almost constant flux, over a longer period (120 250 min) membrane flux was maintained at 30 35 L/(m $\left.{ }^{2} . \mathrm{h}\right)$ between, which is characterized by cross-flow filtration.

Membrane separation processes membrane fouling is inevitable question, flux from the membrane at unit pressure water flux [2000 3000 L/ ( $\left.\left.\mathrm{m}^{2} . \mathrm{h} . \mathrm{M} \mathrm{Pa}\right)\right]$ and the filter $12 \mathrm{~min}$ when [325 
$\mathrm{L} /\left(\mathrm{m}^{2}\right.$.h.M Pa)] compared to the flux seen in about 10 min decreased by $80 \%$, which is caused by impurities in the raw material liquid adsorption membrane, so that the membrane flux decline and affect membrane separation characteristics . To do as much as possible to control membrane fouling factors to improve efficiency, but also requires a certain cleaning method removed to restore the membrane performance, extended service life of pollutants. We can even say that the cleaning film is one of the important factors that determine the effect of membrane separation. Under laboratory conditions, the continuous filtration to flux less than $30 \mathrm{~L} /$ should be cleaned films (so far accumulated per $\mathrm{m}^{2}$ membrane filtration liquid was about $\left.300 \mathrm{~L}\right)\left(\mathrm{m}^{2} . \mathrm{h}\right)$, the extract was used for this experiment medicine contains polysaccharides, gum and other organic matter and calcium sulfate salts, consider using acid, alkali and EDT A as the cleaning agent for cleaning tests.

Single cleaning effect is not ideal, even $0.5 \mathrm{~mol} / \mathrm{L} \mathrm{NaOH}+0.1 \mathrm{~mol} / \mathrm{L}$. EDTA mixture of single cleaning only the flux recovery from $433 \mathrm{~L} /(\mathrm{m} 2 . \mathrm{h} . \mathrm{M} \mathrm{Pa})$ to $1800 \mathrm{~L} /(\mathrm{m} . \mathrm{h}$. MPa ) around; alternately cleaning agent for cleaning a variety of membrane flux recovery is extremely beneficial. In addition, the quality of the film membrane cleaning effect of pollution-related, will increase the difficulty of cleaning severe pollution, the cleaning step increase, prolong the cleaning time. When the membrane cleaning, cleaning agents and cleaning method of selection is determined, we need more experimental work. Since the ceramic membrane performance determines the choice of a wide range of cleaning agents, you can always establish appropriate cleaning methods to recover membrane flux, to ensure that the industrialized microfiltration process.

Ultra-filtration. After ultra-filtration permeate is clear and transparent, the solid content of the permeate dropped to $2.36 \%$, while the solids content of the retentate increased to $3.15 \%$. Illustrated by ultra-filtration, liquid soluble macromolecular impurities have been removed. Process characteristic $20^{\circ} \mathrm{C}$, under the conditions of $0.1 \mathrm{MPa}$, ultra-filtration membrane flux changes with time relationship shown in Figure 4. It is seen from Figure 4, due to pre-microfiltration, ultra-filtration process is quite stable membrane flux attenuation is not significant in the longer period of time (100 400 min) flux individual components stabilized at 35 to $40 \mathrm{~L} /(\mathrm{h}$. MPa) around.

Ultra-filtration membrane cleaning is the key to the success of ultra-filtration applications.

Under laboratory conditions, the flux was filtered to less than $35 \mathrm{~L} /(\mathrm{h}$. MPa) after cleaning the membrane (ultra-filtration membrane filtration thus accumulated to obtain a single liquid about 30 3 L), due to the microfiltration process is removal of the liquid in the micron and submicron suspended impurities, and therefore pollution ultra-filtration membrane is mainly contaminated solute molecules, ions, etc., so the first washed with water, then $0.1 \mathrm{~mol} / \mathrm{L} \mathrm{NaH}$ and $0.05 \mathrm{~mol} / \mathrm{L}$ EDTA mixture of cleaning, washing the membrane flux recovered to $100 \mathrm{~L} /(\mathrm{h}$. MPa), after flux cleaning agent returned to the initial water flux of $250 \mathrm{~L} /(\mathrm{h} . \mathrm{MPa})$ around.

Ultra-filtration membrane cleaning is the key to the success of ultra-filtration applications. Membrane separation process eliminates the alcohol precipitation process of multi-channel processes, to achieve the purpose of removing impurities, still maintained a traditional Chinese medicine decoction and compound compatibility. Table 1 shows that the active ingredient remains the same, which are terpenes, steroids, saponins, alcohol precipitation reaction is more pronounced. Compared with alcohol precipitation, membrane separation process has the following advantages: (1) Extract dried easily, moisture absorption, add a small vehicle; (2) Save a lot of ethanol and the corresponding recovery equipment; (3) To shorten the production cycle, reduction processes and personnel, saving energy.

\section{Conclusion}

Ultra-filtration membrane cleaning is the key to the success of ultra-filtration applications. Membrane separation process of the pharmaceutical preparation is generally unchanged active ingredient; for room temperature operation, no phase change process, test the reaction of certain ingredients than the alcohol precipitation method is more evident in the process and it has its own unique advantages. 
Ceramic membrane cross-flow filtration technology is used to extract medicine directly clarification process effectively removes suspended impurities extract. Those provide a reliable ultra-filtration pretreatment and the characteristics of the ceramic membrane so that it can be easily regenerated.

Ultra-filtration technology is used to remove the liquid in the macromolecular impurities flux attenuation is not significant, stable flux individual components 35 40 L/(h. MPa) around; after using a cleaning agent flux recovered.

Using membrane separation process is technically feasible. Further research will be considered suitable flocculation pretreatment methods to further improve the throughput of the micro filter to adapt to the needs of industrial production; and a separation process for active ingredients in drugs is used to conduct quantitative test.

\section{References}

[1] Lee Soo. Outer Pressure Hollow Fiber Ultra-filtration Membrane Used in the Production of oral Liquid Medicine. The Second Session of the National Film and Membrane Processes Symposium Proceedings, Hangzhou, 1996. 469-470.

[2] Zhang-Yu Z, Li Ran, Li Hong, etc. Ultra-filtration SHL Pin Agent. The Second Session of the National Film and Membrane Processes Symposium Proceedings, Hangzhou, 1996. 459-461.

[3] Guo-You Z, Luo-Ke Q. Microporous Membrane of Hepatic Complex Shu Oral Removing Impurities and Design. Membrane Science and Technology, 1994, 14 (3): 63-67.

[4] Liu-Hong Q, Ren-Cui P, Qu-Ling B. Ultra-filtration Oral ShengmaiYin. Water Processing Technology, 1995, 21 (2): 95-98. 